\title{
Flicking the switch on a molecular gate
}

Electric fields change the permeability of a metal organic framework membrane

By Jorge Gascon

King Abdullah University of Science and Technology (KAUST), KAUST Catalysis Center, Advanced Catalytic Materials, Thuwal 23955-6900, Saudi Arabia.Email: jorge.gascon@kaust.edu.sa

The use of external stimuli to manipulate the properties of well-defined materials may find applications in on-demand drug delivery, separation of molecules, sensing, smart coatings, or artificial tissues (1). Most of these applications rely on changes in the shape, stiffness, pore size, or other properties of soft materials in response to external pressure, temperature changes, or electric or magnetic fields. Despite the success of polymeric materials, because of their low ordered nature, achieving a high resolution into the degree at which these changes occur is not straightforward. On page $x x x$ of this issue, Knebel et al. instead manipulate metal organic framework (MOF) membranes, showing that the membranes can change their pore size upon exposure to an external electrical field, enabling precise separation of different gas molecules. MOFs are formed from a combination of organic ligands and metal nodes or clusters. In some of these materials, host-guest interactions respond to physical and chemical stimuli, leading to breathing or swelling. In other cases, linker rotation or net sliding, which are not necessarily accompanied by crystallographic phase transitions, also provide structural flexibility (3). Because of these structural features, sharp molecular sieving-the separation of two molecules with close kinetic diameters based on size exclusion-has only been demonstrated in a few cases $(4,5)$. Zeolitic imidazolate frameworks (ZIFs), a subclass of MOFs containing imidazolate building units, are an example of soft MOF crystals in which linker rotation plays an important role. Most ZIFs can accommodate bigger molecules in their pores than one would predict based on structural data. For example, ZIF-7, has a crystallographic window size of circa $0.3 \mathrm{~nm}$, yet can accommodate hydrocarbons with kinetic diameters above $0.5 \mathrm{~nm}$. Similarly, ZIF- 8 , the material studied by Knebel et al., has a pore diameter of $0.34 \mathrm{~nm}$, but allows the diffusion of molecules such as $\mathrm{n}$ hexane, benzene, and 1,3,5-trimethylbenzene (over $0.7 \mathrm{~nm}$ in size) $(6,7)$. Many studies have investigated the mechanisms behind MOF flexibility, but efforts to control this property on demand using non-invasive stimuli have been unsuccessful.

Knebel et al. now show that structure polarization due to the application of an external electric field is a non-destructive and reversible way to control gas transport through polycrystalline ZIF8 membranes. The authors report that exposure of ZIF-8 to an external E-field of $500 \mathrm{~V} / \mathrm{mm}$ causes lattice polarization, followed by a crystal structure transition from cubic to monoclinic and triclinic polymorphs. The crystallographic pore aperture in the cubic structure is smaller $(0.34$ $\mathrm{nm})$ than in the polymorphs $(0.36 \mathrm{~nm})$. However, restricted linker mobility in the latter leads to lower fluxes through the membrane and to a much clearer size exclusion in the separation of propylene from propane, even when only circa $30 \%$ of the ZIF-8 undergoes this phase transition under the applied electric field.

Additional experiments performed on non-flexible HKUST-1 membranes demonstrate that electric fields only influence MOFs that display soft mode phase changes. However, many MOFs do behave as soft crystals (8), and the library of materials that could be electrically actuated is 
huge. For instance, several polymorphs are known for the above mentioned ZIF-7, with a strong dipolar linker (9). Besides, the reported findings may only be the beginning. Knebel et al. suggest that if a sufficiently strong electric field is applied, complete linker alignment could be achieved. Should this be the case, we would be one step closer to making molecular gates that are straightforward to actuate.
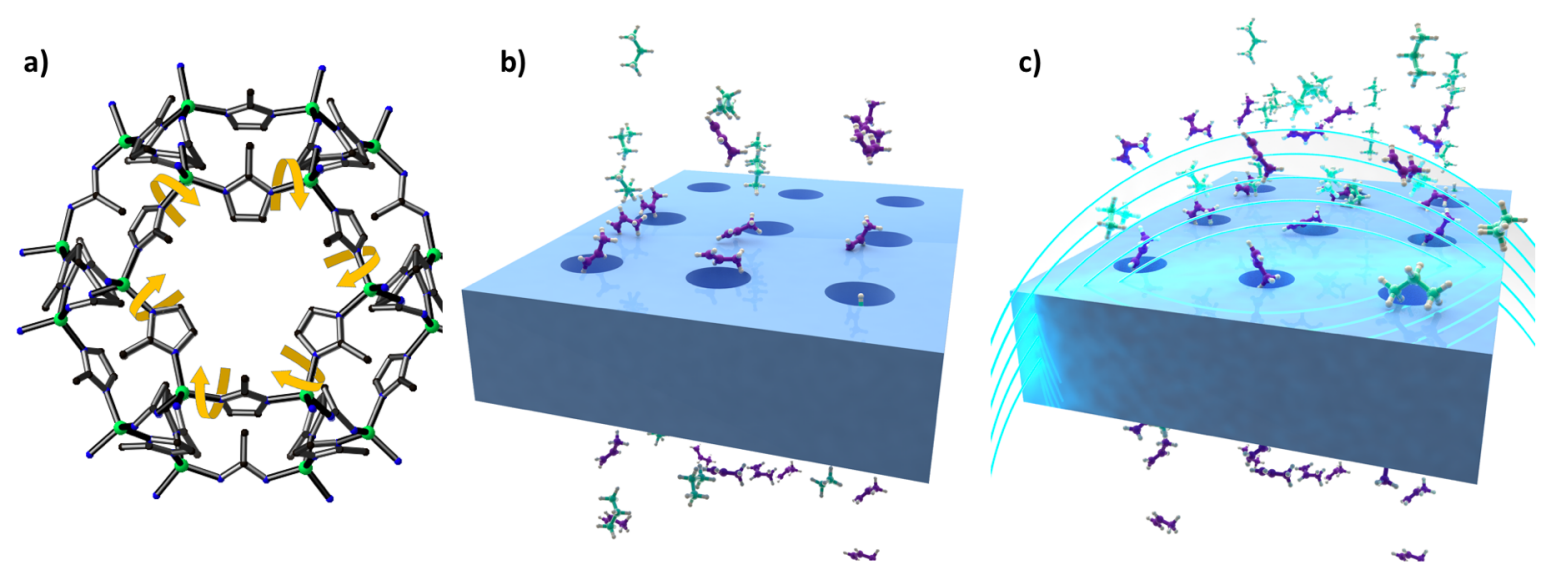

Figure 1: a) the structure of the main SOD cage in ZIF-8. Rotation of the methyl imidazole linkers, indicated by the yellow arrows, allows for the diffusion of molecules bigger than the crystallographic pore size and to moderate selectivities in the separation of propane from propylene (b). Upon exposure to an E-Field (c), lattice polarization restricts linker mobility, resulting in an increase in separation selectivity.

Knebel et al.'s findings may have implications far beyond membrane separation. Although previously suggested in a theoretical study (10), the experimental proof that the crystal structure of MOFs can be manipulated with electric fields opens the door to many other applications. On the basis of the study by Knebel et al., one can start dreaming of very interesting new applications. From adsorptive processes on dynamic beds where pore-size is controlled as a function of gas phase composition to catalysts where selectivity could be tuned for certain substrates by changing the coordination environment of the active site or even electrically actuated artificial organs and tissues.

\section{REFERENCES}

1. M. Wei, Y. Gao, X. Li, M. J. Serpe, Polym. Chem. 8, 127 (2017).

2. A. Knebel et al., Science XXX, XXX (2017).

3. A. Schneemann et al., Chem Soc Rev. 43, 6062 (2014).

4. A. Cadiau et al., Science. 356, 731 (2017).

5. A. Cadiau, K. Adil, P. M. Bhatt, Y. Belmabkhout, M. Eddaoudi, Science. 353, 137 (2016).

6. C. Gucuyener, J. Van Den Bergh, J. Gascon, F. Kapteijn, J Am Chem Soc. 132, 17704 (2010). 
7. K. Zhang et al., J. Phys. Chem. Lett. 4, 3618 (2013).

8. T. D. Bennett, A. K. Cheetham, A. H. Fuchs, F.X. Coudert, Nature chemistry. 9, 11-16 (2017).

9. $\quad$ P. Zhao et al., Chem Mater. 26, 1767 (2014).

10. A. Ghoufi, K. Benhamed, L. Boukli-Hacene, G. Maurin, ACS Cent. Sci. 3, 394 (2017). 